\section{Darmkrebs: Verkürzt Muskelschwund das Leben?}

\begin{abstract}
Aus Querschnittstudien ist bekannt, dass bei Darmkrebs-Patienten eine verringerte Muskelmasse mit einer Erhöhung von Mortalität und Toxizität der Chemotherapie assoziiert ist. Eine Langzeitstudie gab es dazu bisher nicht.
\end{abstract}

$\mathrm{N}$ iederländische Onkologen prüften deshalb in einer prospektiven Studie, wie sich die Muskelmasse während der Chemotherapie verändert und welchen Effekt das auf das Überleben hat. Mithilfe von Computertomografie(CT)-Aufnahmen des Abdomens in Höhe der Lumbalregion L3 ermittelten sie bei 67 Patienten mit metastasiertem Kolorektalkarzinom die Skelettmuskelmasse vor Therapiebeginn und nach median $78 \mathrm{Ta}-$ gen.

Im Mittel waren die Teilnehmer 66,4 Jahre alt (63\% Männer). 78 \% der Patienten erhielten eine Erstlinien-Chemotherapie. $66 \%$ der Patienten erhielten eine Kombination aus Capecitabin und Oxaliplatin mit oder ohne Bevacizumab. Zu Studienbeginn waren $55 \%$ übergewichtig, $8 \%$ adipös. $57 \%$ hatten einen nied- rigen Skelettmuskelindex (SMI; Quotient aus Skelettmuskelfläche auf Höhe L3 und Körperlänge). Die Skelettmuskelfläche nahm bei beiden Geschlechtern innerhalb von drei Monaten um 6,1\% $(\mathrm{p}<0,001) \mathrm{ab}$, entsprechend einer $\mathrm{Ge}$ wichtsreduktion von $1,7 \mathrm{~kg}$ bei Männern und $1,1 \mathrm{~kg}$ bei Frauen. Median betrug das Gesamtüberleben (OS) bei Patienten mit Erstlinientherapie 17,5 Monate und bei Patienten mit einer Zweitlinientherapie 8,5 Monate.

Der Cut-off-Wert beim Muskelschwund betrug 9\%: Werte darüber waren im Vergleich zu niedrigeren Werten mit einem signifikant kürzeren OS assoziiert: Von den Patienten mit Muskelschwund $\geq 9 \%$ lebten nach einem halben Jahr noch $33 \%$ gegeüber $69 \%$ der Patienten mit Werten $<9 \%$; nach einem Jahr waren es $17 \%$ versus $49 \%$ der Patienten. Der unabhängige Zusammenhang von Muskelschwund und OS war selbst dann sichtbar, wenn die Ärzte bei der Berechnung unter anderem Geschlecht, Alter, L-Lactatdehydrogenase(LDH)-Werte vor Therapie und die Tumorprogression bei der ersten CT-Evaluierung berücksichtigten (Hazard Ratio 4,47, 95\%-Konfidenzintervall 2,21-9,05; $\mathrm{p}<0,01)$. Einen Zusammenhang zwischen niedrigem SMI vor der Therapie und Überleben entdeckten die Ärzte nicht.

Fazit: Bei Patienten mit einem metastasierten kolorektalen Karzinom ist Muskelschwund während der palliativen Chemotherapie mit einem verringerten Gesamtüberleben assoziiert. Bei mehr als 9\% Verlust an Muskelmasse leben die Patienten signifikant kürzer, wie die Ergebnisse der prospektiven Studie vermuten lassen.

Peter Leiner

Blauwhoff-Buskermolen S et al. Loss of Muscle Mass During Chemotherapy Is Predictive for Poor Survival of Patients With Metastatic Colorectal Cancer. J Clin Oncol. 2016;34(12):1339-44.

\section{Selektive interne Radiotherapie gegen Lebermetastasen bei kolorektalem Karzinom}

\begin{abstract}
Für Patienten mit einem in die Leber metastasierten kolorektalen Karzinom bietet die Kombination aus standardmäßiger Chemotherapie mit der selektiven internen Radiotherapie ein erweitertes Regime in der Erstlinie.
\end{abstract}

K olorektale Karzinome (CRC) bilden Metastasen bevorzugt in der Leber aus. 80-90\% der CRC-Patienten mit Lebermetastaen gelten bei Diagnosestellung als primär nicht resektabel. Von den vielen interventionellen Therapien fand noch keine Eingang in eine große randomisierte Phase-III-Studie. Einen viel versprechenden Ansatz stellt die selektive interne Radiotherapie (SIRT) dar. Per Infusion in die Arteria hepatica gelangen mit Yttrium-90 markierte Mikrosphären gezielt in den mikrovaskulären Plexus des Tumors, wo sie a-Strahlung emittieren. Frühere Studien mit geringen Fallzahlen ließen auf ein längeres Gesamt- und progressionsfreies Überleben (PFS) nach Behandlung mit SIRT und Erstlinientherapie auf Fluoropyrimidin-Basis schließen.

In der randomisierten Phase-III-Studie SIRFLOX wurde die Wirksamkeit und Sicherheit des modifizierten FOLFOX-Protokolls (mFOLFOX6) mit optionaler Zugabe von Bevacizumab mit einem Regime aus mFOLFOX6 plus SIRT in der Erstlinie verglichen. Untersucht wurden 530 chemotherapienaive Patienten mit nicht resektablem, vornehmlich hepatisch metastasiertem CRC. Primärer Endpunkt war die Progressionsfreiheit an intra- und extrahepatischen Loci. Zu den sekundären Endpunkten gehörten u. a.: leberbezogenes PFS sowie leberbezogenes und gesamtes Tumoransprechen. Es ergaben sich si- gnifikante Vorteile für die SIRT-Kombination beim sekundären Endpunkt „leberbezogenes PFS“ (median 20,5 vs. 12,6 Monate; Hazard Ratio 0,69; $\mathrm{p}=0,002$ ). Das um die Radioembolisation erweiterte Chemotherapieregime steigerte auch das mediane objektive Ansprechen in der Leber von $69 \%$ auf $79 \%$ und die Komplettremissionsrate in der Leber um den Faktor 3 (6,0 vs. 1,9\%). Es fand sich hingegen kein signifikanter Unterschied im gesamten PFS (primärer Endpunkt).

Fazit: Bei Patienten mit einem vornehmlich in die Leber metastasierten CRC verbessert die Kombination aus Chemotherapie und SIRT in der Erstlinie nicht das gesamte PFS. Das Kombinationsregime verlängert jedoch signifikant die Zeitspanne bis zur Progression in der Leber.

Wolfgang Zimmermann

van Hazel GA et al. SIRFLOX: randomized phase III trial comparing first-line mFOLFOX6 (plus or minus bevacizumab) versus mFOLFOX6 (plus or minus bevacizumab) plus selective internal radiation therapy in patients with metastatic colorectal cancer. J Clin Oncol. 2016;34(15):1723-31. 